\title{
Des PME essentielles pour une croissance plus inclusive
}

Écrit par : Angel Gurría, Secrétaire général de l'OCDE

Dernière mise à jour : 8 février 2018

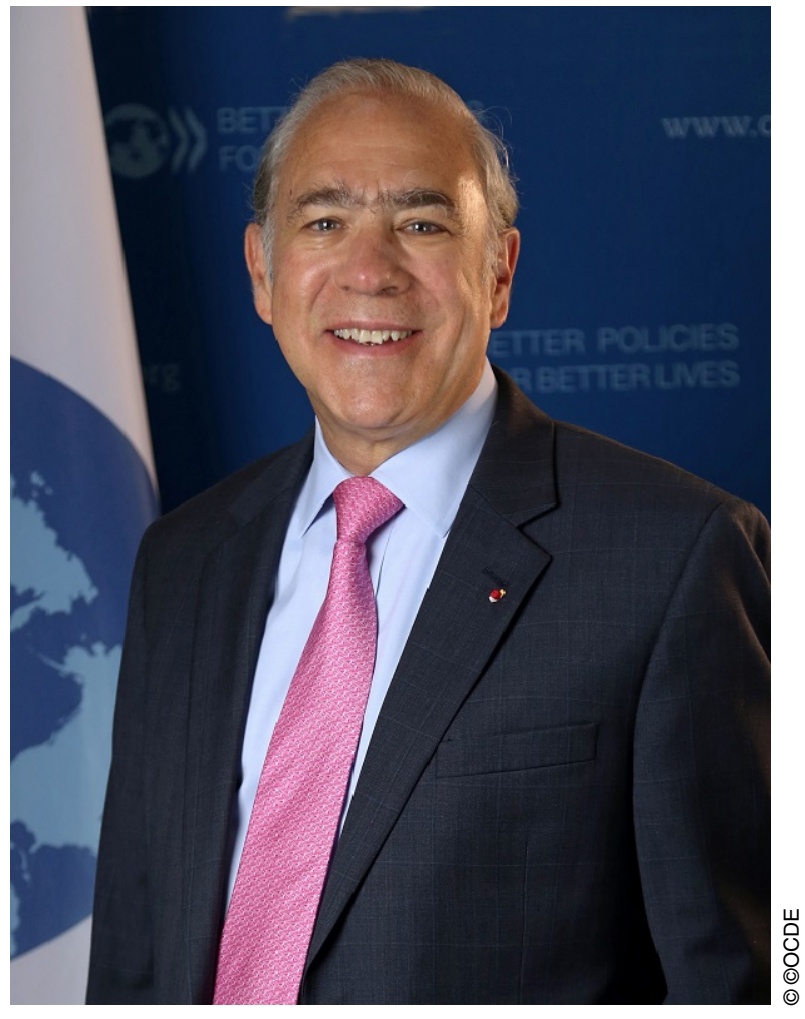

Des PME essentielles pour une croissance plus inclusive

Les petites et moyennes entreprises (PME) jouent un rôle majeur, crucial dans bien des cas, dans nos économies et dans nos sociétés. Dans les pays de l'OCDE, $99 \%$ environ des entreprises sont des PME. Principales pourvoyeuses d'emplois, elles concentrent environ $60 \%$ de l'emploi dans le secteur manufacturier et $75 \%$ dans celui des services, et sont à l'origine, en moyenne, de 50 à $60 \%$ de la création de valeur ajoutée. Dans les économies émergentes, elles représentent jusqu'à $45 \%$ de l'emploi total et $33 \%$ du PIB. 
Les nouvelles petites entreprises se caractérisent souvent par leur dynamisme et leur capacité d'innovation. Les PME européennes comptent ainsi pour $20 \%$ des dépôts de brevets dans les domaines liés aux biotechnologies. Et plus des deux tiers des champions nationaux de la productivité en Europe - les entreprises les plus productives dans leur pays et au sein de leur secteur d'activité - sont des PME, une proportion qui dépasse $80 \%$ dans les services à forte intensité de savoir.

La bonne santé du secteur des PME est donc un facteur essentiel d'une croissance inclusive, en favorisant la création d'emplois dans toutes les régions et tous les secteurs, y compris pour les personnes peu qualifiées. Les PME contribuent au renforcement des compétences, ainsi qu'à l'accès aux soins et aux services sociaux de leurs salariés. Elles sont également un formidable instrument de lutte contre le fléau de l'économie informelle.

Toutefois, la situation des PME est contrastée et compliquée. À l'échelle de l'OCDE, le taux de productivité des microentreprises est ainsi deux fois moins élevé environ que celui des autres PME, qui est lui-même inférieur à celui des grandes entreprises. L'écart de productivité s'est également creusé depuis la crise mondiale, notamment dans le secteur manufacturier, accentuant les inégalités sociales et de revenu.

De plus, l'incertitude, la complexité et l'incohérence réglementaires affectent bien davantage les PME que les autres entreprises. Les coûts engendrés par le respect de la réglementation et la discipline fiscale sont parfois excessifs, et contribuent à alimenter l'économie informelle. Ainsi, les petites entreprises qui cherchent à croître ou à se lancer dans des activités à plus forte valeur ajoutée peuvent avoir l'impression que cela n'en vaut pas la peine. Il en résulte un engrenage où la faible productivité se combine à un niveau élevé d'activité souterraine.

De nombreuses PME se heurtent aussi à des obstacles pour obtenir des financements. Le Tableau de bord de l'OCDE sur le financement des PME et des entrepreneurs 2018 fait état d'un recul de 4,1 \% en glissement annuel des nouveaux prêts aux PME au Royaume-Uni en 2016. Aux Pays-Bas, la baisse s'établit à 17,1\%. L'Amérique latine fait face à des défis similaires. Selon les auteurs du rapport de l'OCDE Perspectives économiques de l'Amérique latine 2017, les PME de la région n'ont reçu que $12 \%$ seulement du volume total des crédits octroyés, alors que ce pourcentage s'élève à $25 \%$ dans les pays de l'OCDE. De fait, un tiers des petites entreprises d'Amérique latine citent l'accès au financement comme une sérieuse entrave à leur développement.

À l'heure où l'économie mondiale affiche des performances toujours décevantes, il est essentiel de favoriser l'innovation et le développement des PME pour stimuler la productivité globale et permettre une croissance plus inclusive et durable. Toutefois, cela dépendra dans une large mesure de la capacité de ces entreprises à surfer sur la vague du numérique. 
Trop de PME ne profitent pas des possibilités de la révolution numérique et de la mondialisation. Celles qui n'auront pas su prendre la vague du numérique finiront, tôt ou tard, par se laisser distancer, sans aucun moyen de revenir dans la course. La transformation numérique de l'économie est une sorte de "mondialisation sous stéroïdes ", amenant des changements rapides et d'une ampleur sans précédent. Cette révolution ouvre de formidables perspectives aux PME. Les technologies numériques favorisent l'émergence de petites entreprises qui, d'emblée, ont une "dimension mondiale », et donnent aux PME installées de nouvelles possibilités de se développer, de renforcer leur compétitivité et de devenir des acteurs de l'économie mondiale.

Cette numérisation de l'économie pose également de sérieux défis. De nombreuses PME peinent à exploiter le potentiel du numérique pour améliorer leur accès aux marchés et accroître leur productivité. Dans les pays de l'OCDE, 20 $\%$ seulement des PME sont présentes dans le commerce en ligne, contre $40 \%$ des grandes entreprises. Pour combler ce retard, des investissements sont nécessaires dans plusieurs domaines : renforcement des compétences, changements organisationnels, innovation de procédé, nouveaux systèmes et modèles économiques, autant de facteurs indispensables pour encourager l'adoption et l'utilisation efficace des technologies numériques.

Les chaînes de valeur mondiales offrent aussi aux PME de nouvelles possibilités de participer à l'économie mondiale. La participation aux marchés mondiaux demeure cependant inégale au sein de cette population d'entreprises. Dans les pays de l'OCDE, $15 \%$ des microentreprises en moyenne participent aux échanges internationaux, à comparer aux $60 \%$ de petites entreprises et aux $80 \%$ d'entreprises de taille moyenne. Les PME sont en outre davantage touchées par les obstacles aux échanges. Ainsi, dans le domaine de l'exportation transfrontalière de services, un niveau moyen de restrictivité se traduit par des droits de douane supérieurs de $12 \%$ à ceux supportés par les grands groupes.

L'OCDE œuvre aux côtés des pouvoirs publics pour aider les PME à relever ces défis, à tirer parti de la révolution numérique et à s'imposer comme des acteurs majeurs sur la scène économique mondiale. L'élaboration d'une stratégie de l'OCDE en faveur des PME est actuellement à l'étude. C'est d'ailleurs une nécessité et, à vrai dire, l'Organisation n'a que trop tardé. Il est temps de rattraper ce temps perdu et de devenir la « référence incontournable » sur les politiques à l'égard des PME.

Cet éditorial s'inspire de l'allocution prononcée par M. Angel Gurría, Secrétaire général de l'OCDE, à l'occasion de l'ouverture de la Conférence ministérielle de l'OCDE sur les PME qui s'est tenue à Mexico le 22 février 2018.

L'intégralité du discours (1 300 mots) est disponible à l'adresse https://oe.cd/2kZ 
Presentation of the 2018 OECD SME Finance Scoreboard: http://www.oecd.org/ industry/launch-of-oecd-sme-finance-scoreboard-mexico-2018.htm

Twitter:@A_Gurria

www.oecdobserver.org/angelgurria

www.oecd.org/about/secretary-general

@L'Observateur de l'OCDE, T1 2018 